\title{
Effects of immediate versus delayed frozen embryo transfer in high responder patients undergoing freeze-all cycles
}

\author{
Na Zuo', Yingzhuo Gao', Ningning Zhang ${ }^{2}, \mathrm{Da} \mathrm{Li}^{1^{*}}$ and Xiuxia Wang ${ }^{1 *}$
}

\begin{abstract}
Background: Frozen embryo transfer (FET) can greatly improve the pregnancy outcomes for high responder patients. However, it is not known whether the timing of FET is a risk factor on pregnancy outcomes in high responder patients undergoing freeze-all cycles.
\end{abstract}

Methods: A retrospective cohort study to compare the pregnancy outcomes of the immediate and delayed FET groups in high responder patients undergoing freeze-all cycles. The two groups were defined as that FET took place either within the first menstrual cycle following oocyte retrieval or afterwards. Propensity score matching was used to make the potential risk factors of the two groups comparable. Multivariable regression analysis was used to study the effect of the timing of FET on pregnancy outcomes in the entire cohort and propensity score-matched cohort, even in different controlled ovarian hyperstimulation protocol cohorts as subgroup analysis.

Results: We obtained 1130 patients in immediate FET group and 998 patients in delayed FET group, and the average age of the two groups were 30.30 and 30.63. We showed that the immediate FET group were equivalent to delayed FET group in the entire cohort [clinical pregnancy rate (CPR), $61.0 \%$ versus $63.4 \%$, adjusted odd ratio (OR), 0.939, 95\% confidence interval (CI), 0.781-1.129; spontaneous abortion rate (SAR), $10.1 \%$ versus 12.6\%, adjusted $\mathrm{OR}, 0.831,95 \% \mathrm{Cl}(0.628-1.098)$; live birth rate (LBR), 49.9\% versus 49.2\%, adjusted OR, 1.056, 95\% Cl (0.883-1.263)]. The same results were obtained by $x^{2}$ test in the propensity score-matched cohort (CPR, 60.5\% versus 63.5\%; SAR, $11.6 \%$ versus $12.3 \%$; $L B R, 48 \%$ versus $49.3 \%)(P>0.05)$. Subgroup analysis indicated that pregnancy outcomes of immediate FET were no difference to delayed FET in gonadotropin-releasing hormone agonist (GnRH-a) protocol $(P>0.05)$. The SAR of the immediate FET group were lower than that of the delayed FET group in GnRH antagonist protocol (adjusted OR, 0.645, 95\% Cl, 0.430-0.966) $(P<0.05)$, no differences were observed in CPR and LBR $(P>0.05)$.

Conclusions: The pregnancy outcomes of immediate FET were no difference to delayed FET in high responder population undergoing freeze-all cycles.

Keywords: High ovarian response, Frozen embryo transfer, Timing, GnRH analogue, In vitro fertilization, Pregnancy outcomes

\footnotetext{
*Correspondence: leeda@ymail.com; wangxxsj@sina.cn

${ }^{1}$ Center of Reproductive Medicine, Shengjing Hospital of China Medical University, Shenyang 110004, China

Full list of author information is available at the end of the article
}

(c) The Author(s). 2021 Open Access This article is licensed under a Creative Commons Attribution 4.0 International License, which permits use, sharing, adaptation, distribution and reproduction in any medium or format, as long as you give appropriate credit to the original author(s) and the source, provide a link to the Creative Commons licence, and indicate if changes were made. The images or other third party material in this article are included in the article's Creative Commons licence, unless indicated otherwise in a credit line to the material. If material is not included in the article's Creative Commons licence and your intended use is not permitted by statutory regulation or exceeds the permitted use, you will need to obtain permission directly from the copyright holder. To view a copy of this licence, visit http://creativecommons.org/licenses/by/4.0/ The Creative Commons Public Domain Dedication waiver (http://creativecommons.org/publicdomain/zero/1.0/) applies to the data made available in this article, unless otherwise stated in a credit line to the data. 


\section{Background}

Controlled ovarian hyperstimulation $(\mathrm{COH})$ is the key step in in vitro fertilization and embryo transfer (IVFET). High ovarian response (HOR) refers to the abnormal sensitivity of the ovary to gonadotropin, which leads to simultaneous development of multiple follicles and increases the risk of ovarian hyperstimulation syndrome (OHSS) [1]. Supraphysiological steroid hormones during $\mathrm{COH}$ affect endometrial receptivity by changing the endometrial immune environment and gene expression [2-4], resulting in poor pregnancy outcomes [5]. Adopting a freeze-all strategy in HOR patients can greatly reduce the risk of OHSS and avoid the influence of $\mathrm{COH}$ on endometrial receptivity [6]. An increasing number of studies have confirmed that frozen embryo transfer (FET) has better pregnancy and perinatal outcomes than fresh embryo transfer [7-9].

However, the best time to perform FET following $\mathrm{COH}$ in HOR patients is controversial in clinical work. Postponement of FET may increase the anxiety of patients [10]; in the immediate FET cycle, poor endometrial receptivity or physical condition may not be fully recovered to the pre-stimulation state, which may affect pregnancy outcomes [11]. It is unclear whether the detrimental effects on endometrial receptivity caused by $\mathrm{COH}$ would be sustained over a long period of time, up to the subsequent menstrual cycle, especially in patients with HOR who are most affected by $\mathrm{COH}$. Moreover, the use of different gonadotropin-releasing hormone $(\mathrm{GnRH})$ analogues in the process of $\mathrm{COH}$ act on the pituitary in different ways [12], and it is controversial whether the timing of FET affects pregnancy outcomes in different $\mathrm{COH}$ protocols $[11,13]$.

Thus, this study aimed to investigate whether the FET timing affects pregnancy outcomes in HOR patients undergoing freeze-all cycles, whether different $\mathrm{COH}$ protocols affect pregnancy outcomes in the subsequent FET cycle, and to provide reference results for the HOR population to choose the optimal time to start FET.

\section{Materials and methods}

\section{Study population and design}

We conducted a retrospective cohort study including all patients from January 2015 to March 2019 at our reproductive medicine center and the study was conducted in accordance with ethical standards (2020PS006F); informed consent was obtained from all subjects. The inclusion criteria were as follows: (1) patients on their first IVF or intracytoplasmic sperm injection (ICSI) cycle who were diagnosed with $\mathrm{HOR}$ and adopted a freeze-all strategy. The diagnostic criteria for HOR was $>5000 \mathrm{pg} /$ $\mathrm{ml}$ of estradiol on human chorionic gonadotropin (HCG) day or more than 15 oocytes retrieved [14, 15]; (2) three different selected $\mathrm{GnRH}$ analogues stimulation protocols, including short-acting $\mathrm{GnRH}$ agonist (GnRHa) long protocol, long-acting $\mathrm{GnRH}$-a long protocol, and GnRH antagonist (GnRH-ant) protocol; (3) women 2045 years old; and (4) hormone replacement therapy (HRT) for endometrial preparation in the FET cycle. The exclusion criteria were as follows: (1) presence of uterine abnormalities; (2) patients with endometriosis and adenomyosis; (3) presence of autoimmune, endocrine, and metabolic diseases; (4) previous diagnosis of uterine adhesion; (5) patients with chromosomal abnormalities; (6) patients who underwent blastocyst biopsy for pre-implantation genetic testing; (7) patients using frozen donor semen; (8) patients using long-acting GnRH-a as pretreatment for FET after the freeze-all cycle; (9) patients with a natural cycle for endometrial preparation for FET; (10) patients with no embryo formation for FET; and (11) patients with ectopic pregnancies.

Patients were divided into the immediate FET group and the delayed FET group, which were defined as FET that took place within the first menstrual cycle following oocyte retrieval or afterwards.

\section{Ovarian stimulation protocol, endometrial preparation protocol, and luteal support}

According to age, anti-Müllerian hormone, body mass index (BMI), number of antral follicles in bilateral ovaries, and prior response to stimulation, we can predict the HOR population and determine the initial dose of gonadotropin to prevent the occurrence of OHSS [16, 17]. All patients were treated with the following three $\mathrm{COH}$ protocols: the short-acting $\mathrm{GnRH}$-a long protocol involved daily subcutaneous injection of $0.05 \mathrm{mg}$ of short-acting GnRH-a triptorelin (Diphereline, $0.1 \mathrm{mg}$, IPSEN, France) at the middle luteal phase of the menstrual cycle as pituitary down-regulation for 14 days, and gonadotropin introduction at the subsequent menstruation; the long-acting GnRH-a long protocol involved a single-dose intramuscular injection of a quarter to a full dose $(0.75-3.75 \mathrm{mg}$ ) of long-acting GnRH-a (Diphereline, $3.75 \mathrm{mg}$, IPSEN, France) on the second day of menstruation, with gonadotropin given 20-30 days later when the follicle diameter reached $3-5 \mathrm{~mm}$; the flexible $\mathrm{GnRH}$-ant protocol involved starting gonadotropin on the second day of menstruation, and GnRH antagonist (Cetrotide, Merck Serono, France) was added when the lead follicle reached $13-14 \mathrm{~mm}$ in diameter or when the estradiol was $>300 \mathrm{pg} / \mathrm{ml}$. Follicle development was detected by transvaginal ultrasonography, and the dosage of gonadotropin was adjusted according to the different ovarian responses.

When the follicles reached a mean diameter of $>17$ $\mathrm{mm}$, final oocyte maturation was triggered. HCG or triptorelin was used alone or in combination in the GnRH- 
ant protocol. Only HCG was used for the trigger in the $\mathrm{GnRH}$-a long protocol. Oocyte retrieval was performed $36 \mathrm{~h}$ after triggering by transvaginal ultrasound-guided aspiration. Cleavage stage embryo quality was evaluated at day three based on the Istanbul consensus to confirm extended embryo culture, and the day three embryos with a cell number of $\geq 7$ and fragmentation of $<20 \%$ were graded as good quality [18]. Blastocyst morphology was evaluated in the morning of days five and six according to the Gardner criteria [19], and only blastocysts scoring $4 \mathrm{BB}$ or higher were graded as good quality. We adopted a freeze-all strategy for HOR patients to avoid

Table 1 Baseline characteristics of immediate and delayed FET groups in the entire and propensity score-matched cohorts

\begin{tabular}{|c|c|c|c|c|c|c|}
\hline \multirow[t]{2}{*}{ Potential risk factors } & \multicolumn{2}{|l|}{$\begin{array}{l}\text { Entire cohort } \\
(n=2128)\end{array}$} & \multirow[t]{2}{*}{$\begin{array}{l}P \text { - } \\
\text { value }\end{array}$} & \multicolumn{2}{|c|}{$\begin{array}{l}\text { Propensity score-matched cohort* } \\
(n=1366)\end{array}$} & \multirow[t]{2}{*}{$\begin{array}{l}P \text { - } \\
\text { value }\end{array}$} \\
\hline & $\begin{array}{l}\text { Immediate } \mathrm{FET}^{\mathrm{a}} \\
(n=1130)\end{array}$ & $\begin{array}{l}\text { Delayed FET } \\
(n=998)\end{array}$ & & $\begin{array}{l}\text { Immediate } \mathrm{FET}^{\mathrm{a}} \\
(n=683)\end{array}$ & $\begin{array}{l}\text { Delayed } \mathrm{FET}^{\mathrm{b}} \\
(n=683)\end{array}$ & \\
\hline Maternal age (years) & & & 0.455 & & & 1.000 \\
\hline$\leq 34$ & $976(86.4)$ & $851(85.3)$ & & $622(91.1)$ & $622(91.1)$ & \\
\hline $35-37$ & $114(10.1)$ & $101(10.1)$ & & $48(7.0)$ & $48(7.0)$ & \\
\hline$\geq 38$ & $40(3.5)$ & $46(4.6)$ & & $13(1.9)$ & $13(1.9)$ & \\
\hline BMI $\left(\mathrm{kg} / \mathrm{m}^{2}\right)$ & & & 0.431 & & & 1.000 \\
\hline$<18.5$ & $70(6.2)$ & $58(5.8)$ & & $465(68.1)$ & $465(68.1)$ & \\
\hline $18.5-24.9$ & $750(66.4)$ & $641(64.2)$ & & $22(3.2)$ & $22(3.2)$ & \\
\hline$\geq 25$ & $310(27.4)$ & $299(30.0)$ & & $196(28.7)$ & $196(28.7)$ & \\
\hline Insemination method & & & 0.056 & & & 1.000 \\
\hline IVF & $814(72.0)$ & $681(68.2)$ & & $490(71.7)$ & $490(71.7)$ & \\
\hline ICSI & $316(28.0)$ & $317(31.8)$ & & $193(28.3)$ & $193(28.3)$ & \\
\hline $\mathrm{COH}$ protocol & & & 0.000 & & & 1.000 \\
\hline GnRH-ant protocol & $485(42.9)$ & $433(43.4)$ & $0.273^{\#}$ & $284(41.6)$ & $284(41.6)$ & $0.651^{\#}$ \\
\hline HCG trigger & $330(68.0)$ & $306(70.7)$ & & $199(70.1)$ & $199(70.1)$ & \\
\hline $\mathrm{GnRH}-\mathrm{a}$ trigger & $137(28.2)$ & $105(24.2)$ & & $79(27.8)$ & $79(27.8)$ & \\
\hline Double trigger & $18(3.7)$ & $22(5.1)$ & & $6(2.1)$ & $6(2.1)$ & \\
\hline Short acting GnRH-a protocol & $566(50.1)$ & $434(43.5)$ & & $335(49.0)$ & $335(49.0)$ & \\
\hline Long acting GnRH-a protocol & $79(7.0)$ & $131(13.1)$ & & $64(9.4)$ & $64(9.4)$ & \\
\hline Number of embryo transfer & & & 0.000 & & & 1.000 \\
\hline 1 & $265(23.5)$ & $416(41.7)$ & & $208(30.5)$ & $208(30.5)$ & \\
\hline 2 & $865(76.5)$ & $582(58.3)$ & & $475(69.5)$ & $475(69.5)$ & \\
\hline Embryo stage & & & 0.000 & & & 1.000 \\
\hline Cleavage stage & $711(62.9)$ & $499(50.0)$ & & $418(61.2)$ & $418(61.2)$ & \\
\hline Blastocyst stage & $419(37.1)$ & $499(50.0)$ & & $265(38.8)$ & $265(38.8)$ & \\
\hline Top or good quality embryo transfer & $947(83.3)$ & $838(84.0)$ & 0.919 & $584(85.5)$ & $573(83.9)$ & 0.408 \\
\hline \multicolumn{7}{|l|}{ Cause of infertility } \\
\hline Tubal factor & $720(63.7)$ & $630(63.1)$ & 0.778 & $456(66.8)$ & $456(66.8)$ & 1.000 \\
\hline Ovulatory disorder & $304(26.9)$ & $257(25.8)$ & 0.548 & $167(24.5)$ & $167(24.5)$ & 1.000 \\
\hline Male factor & $452(40.0)$ & $420(42.1)$ & 0.329 & $273(40.0)$ & $273(40.0)$ & 1.000 \\
\hline Unexplained factor & $48(4.2)$ & $46(4.6)$ & 0.686 & $16(2.3)$ & $16(2.3)$ & 1.000 \\
\hline Multiple pregnancies & $216(19.1)$ & 137 (13.7) & 0.001 & 106 (15.5) & $112(16.4)$ & 0.658 \\
\hline
\end{tabular}

Abbreviations: $\mathrm{BMI}$, body mass index; $\mathrm{COH}$, controlled ovarian hyperstimulation; FET, frozen embryo transfer; GnRH-a, gonadotrophin-releasing hormone agonist; $\mathrm{GnRH}$-ant, gonadotrophin-releasing hormone antagonist; HRT, hormone replacement therapy; IVF, in vitro fertilization; ICSI, intracytoplasmic sperm injection. Data are presented as number (\%)

${ }^{\mathrm{a}}$ Immediate FET and ${ }^{\mathrm{b}}$ Delayed FET means FET took place either within the first menstrual cycle following oocyte retrieval or afterwards

*The predictors of propensity score matching were maternal age, BMI, insemination method, $\mathrm{COH}$ protocol, number of embryo transferred, embryo stage, top or good embryo transfer, and cause of infertility. The match tolerance was set to 0.000001 . Categorical variables were compared with $X^{2}$ test. ${ }^{\#} P$ value of differences of different trigger drugs in $\mathrm{GnRH}$-ant protocol 
the occurrence of OHSS. The patients were informed that they could chose the menstrual cycle following oocyte retrieval for FET all by themselves.

Hormone replacement therapy (HRT) was used for endometrial preparation in the FET cycle, with $4-8 \mathrm{mg}$ of estradiol valerate (Progynova, Bayer, Germany) taken orally for at least ten days from the 3rd to 5th day of menstruation to promote the growth of endometrium. Ultrasonic examination should be completed before medication, when the thickness of the endometrium is < $6 \mathrm{~mm}$ and the drug can be used, otherwise the FET in this cycle will be cancelled. Vitrification and warming procedures were performed using Embryo Vitrification/ Thawing Media (KITAZATO) according to the manufacturer's instructions. Cleavage-stage embryos were warmed on the day before transfer, cultured for approximately $24 \mathrm{~h}$, and then transferred. The blastocysts were warmed on the day of transfer, kept in culture for approximately one hour, and then transferred.

The luteal phase was supported by $90 \mathrm{mg}$ per day of vaginal progesterone gel (Crinone, Fleet Laboratories Ltd., UK) administered vaginally, and estradiol was maintained at the original dose. Luteal support was continued until 11 weeks of gestational age.

\section{Statistical analysis}

The primary outcome of our study was live birth rate (LBR). Secondary endpoints were clinical pregnancy rate (CPR) and spontaneous abortion rate (SAR). Clinical pregnancy was defined as the detection of a gestational sac through ultrasound imaging at seven weeks of gestational age [20]. In China, spontaneous abortion was

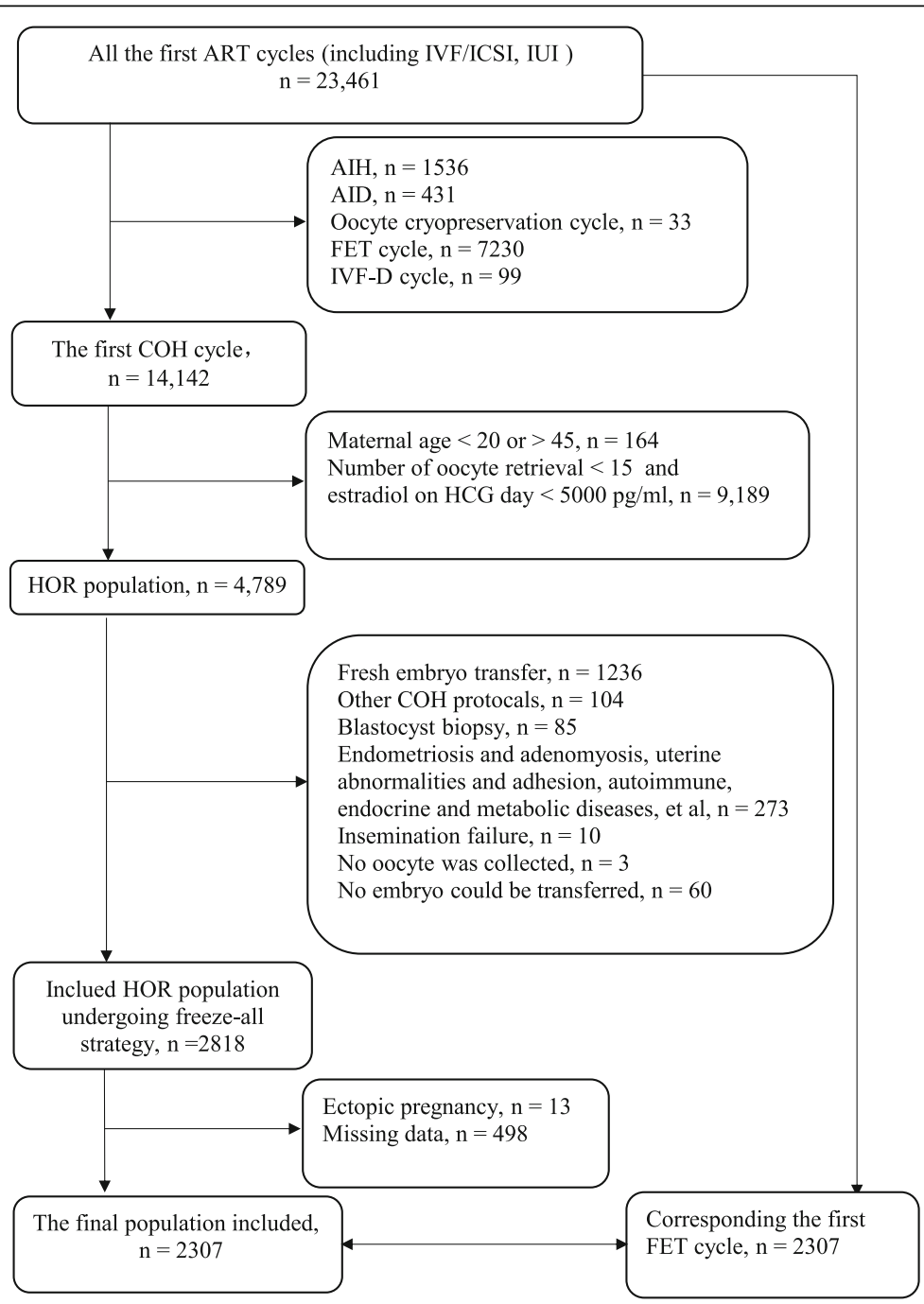

Abbreviations: ART, assisted reproductive technology; $\mathrm{COH}$, controlled ovarian hyperstimulation; FET, frozen embryo transfer; HOR, high ovarian response; IUI, intrauterine insemination; IVF, in vitro fertilization; IVF-D, IVF using frozen donor semen; ICSI, intracytoplasmic sperm injection.

Fig. 1 Flow chat showing the selection of the study polulation 
Table 2 Multivariable logistic regression analysis on potential risk factors for pregnancy outcomes in the entire cohort

\section{Potential risk factor variables}

Clinical pregnancy

FET timing (Immediate FET $^{\mathrm{a}}$ versus delayed $\mathrm{FET}^{\mathrm{b}}$ )

Age $(35-37$ versus $\leq 34)$

Age $(\geq 38$ versus $\leq 34)$

BMI (< 18.5 versus 18.5-24.9)

BMI ( $\geq 25$ versus 18.5-24.9)

$\mathrm{COH}$ protocol (short-acting $\mathrm{GnRH}$-a versus $\mathrm{GnRH}$-ant)

$\mathrm{COH}$ protocol (long-acting $\mathrm{GnRH}$-a versus $\mathrm{GnRH}$-ant)

Trigger type (GnRH-a trigger versus HCG trigger)

Trigger type (Double trigger versus HCG trigger)

Insemination method (ICSI versus IVF)

Number of embryo transferred (2 versus 1)

Embryo stage (blastocyst versus cleavage)

Top or good quality embryo transfer (yes versus no)

Tubal factor (yes versus no)

Ovulatory disorder (yes versus no)

Male factor (yes versus no)

Unexplained factor (yes versus no)

Spontaneous abortion

FET timing (Immediate $\mathrm{FET}^{\mathrm{a}}$ versus delayed $\mathrm{FET}^{\mathrm{b}}$ )

Age $(35-37$ versus $\leq 34)$

Age ( $\geq 38$ versus $\leq 34)$

$\mathrm{BMI}(<18.5$ versus 18.5-24.9)

$\mathrm{BMI}(\geq 25$ versus $18.5-24.9)$

$\mathrm{COH}$ protocol (short-acting $\mathrm{GnRH}$-a versus $\mathrm{GnRH}$-ant)

$\mathrm{COH}$ protocol (long-acting $\mathrm{GnRH}$-a versus $\mathrm{GnRH}$-ant)

Trigger type (GnRH-a trigger versus HCG trigger)

Trigger type (Double trigger versus HCG trigger)

Insemination method (ICSI versus IVF)

Number of embryo transferred (2 versus 1)

Embryo stage (blastocyst versus cleavage)

Top or good quality embryo transfer (yes versus no)

Tubal factor (yes versus no)

Ovulatory disorder (yes versus no)

Male factor (yes versus no)

Unexplained factor (yes versus no)

Live birth

FET timing (Immediate $\mathrm{FET}^{\mathrm{a}}$ versus delayed $\mathrm{FET}^{\mathrm{b}}$ )

Age $(35-37$ versus $\leq 34)$

Age ( $\geq 38$ versus $\leq 34)$

BMI ( $<18.5$ versus 18.5-24.9)

BMI ( $\geq 25$ versus 18.5-24.9)

$\mathrm{COH}$ protocol (short-acting $\mathrm{GnRH}$-a versus $\mathrm{GnRH}$-ant)

$\mathrm{COH}$ protocol (long-acting $\mathrm{GnRH}$-a versus $\mathrm{GnRH}$-ant)
Adjusted $\mathrm{OR}^{*}(95 \% \mathrm{Cl})$

P-value

$0.939(0.781-1.129)$

$0.929(0.690-1.251)$

NS

$0.422(0.268-0.663)$

NS

$P<0.001$

$1.073(0.729-1.577)$

NS

$1.120(0.910-1.378)$

NS

1.256 (1.010-1.562)

$P<0.05$

1.363 (0.973-1.909)

NS

1.267 (0.924-1.738)

NS

2.737 (1.227-6.106)

$P<0.05$

$0.875(0.687-1.115)$

NS

1.573 (1.147-2.156)

$P<0.01$

2.682 (1.999-3.599)

$P<0.001$

1.203 (0.940-1.539)

NS

$1.110(0.877-1.407)$

NS

1.111 (0.877-1.407)

NS

$1.172(0.925-1.486)$

NS

$1.452(0.870-2.423)$

NS

$0.831(0.628-1.098)$

NS

1.088 (0.693-1.707)

NS

$1.214(0.638-2.310)$

NS

0.967 (0.505-1.852)

NS

1.787 (1.336-2.391)

$P<0.001$

$0.833(0.594-1.167)$

NS

$1.236(0.758-2.014)$

NS

0.871 (0.550-1.379)

NS

$1.465(0.642-3.343)$

NS

$1.114(0.768-1.617)$

NS

0.965 (0.624-1.490)

NS

1.200 (0.769-1.810)

NS

$1.114(0.754-1.647)$

NS

0.729 (0.514-1.033)

NS

1.335 (0.949-1.878)

NS

0.709 (0.491-1.022)

NS

$0.744(0.347-1.596)$

NS

$1.056(0.883-1.263)$

NS

$0.936(0.701-1.251)$

NS

$0.365(0.222-0.599)$

$P<0.001$

1.085 (0.748-1.574)

NS

0.841 (0.688-1.028)

NS

1.267 (1.023-1.569)

$P<0.05$

$1.241(0.895-1.720)$ 
Table 2 Multivariable logistic regression analysis on potential risk factors for pregnancy outcomes in the entire cohort (Continued)

\begin{tabular}{lll}
\hline Potential risk factor variables & Adjusted OR* $\mathbf{( 9 5 \% ~ C l )}$ & $P$-value \\
\hline Trigger type (GnRH-a trigger versus HCG trigger) & $1.316(0.968-1.789)$ & NS \\
Trigger type (Double trigger versus HCG trigger) & $1.956(1.002-3.821)$ & NS \\
Insemination method (ICSI versus IVF) & $0.892(0.704-1.129)$ & NS \\
Number of embryo transferred (2 versus 1) & $1.460(1.092-1.953)$ & $P<0.05$ \\
Embryo stage (blastocyst versus cleavage) & $2.289(1.748-2.998)$ & $P<0.001$ \\
Top or good-quality embryo transfer (yes versus no) & $1.224(0.961-1.559)$ & NS \\
Tubal factor (yes versus no) & $1.229(1.032-1.635)$ & $P<0.05$ \\
Ovulatory disorder (yes versus no) & $0.951(0.757-1.195)$ & NS \\
Male factor (yes versus no) & $1.326(1.054-1.668)$ & $P<0.05$ \\
Unexplained factor (yes versus no) & $1.549(0.935-2.518)$ & NS
\end{tabular}

Abbreviations: BMI, body mass index; FET, frozen embryo transfer; $\mathrm{GnRH}$-a, gonadotrophin-releasing hormone agonist; GnRH-ant, gonadotrophin-releasing hormone antagonist; IVF, in vitro fertilization; ICSI, intracytoplasmic sperm injection; OR, odds ratio; Cl, confidence interval; NS, not significant ${ }^{a}$ Immediate FET and ${ }^{b}$ delayed FET means FET took place either within the first menstrual cycle following oocyte retrieval or afterwards

*Using the multivariable logistic regression and adjusting for maternal age, BMI, insemination method, COH protocol, trigger type, number of embryo transferred, embryo stage, top or good quality embryo transfer and cause of infertility

defined as the loss of pregnancy spontaneously after clinical pregnancy and before 28 weeks of gestational age, and live birth was defined as the survival delivery after 28 weeks of gestational age.

As an observational study, multiple maternal and IVF characteristics were considered as potential risk factors that could moderate pregnancy outcomes, and the potential risk factors between the immediate and delayed FET groups were unbalanced distribution (Table 1). Thus, we used propensity score matching (PSM) to make the potential risk factors between the immediate and delayed FET groups balanced and comparable. We used 1:1 nearest-neighbor matching without replacement to compare the variables and tried the match tolerance value from 1 to 0 until $P$ values of the variable between the two groups were 1.000. $X^{2}$ test was performed for comparison of the categorical variables of the immediate and delayed FET groups (Table 1).

As the effect of FET timing on different $\mathrm{COH}$ protocols were controversial, subgroup analysis was performed. Multivariable logistic regression models were calculated on each $\mathrm{COH}$ cohort, with the timing of FET as the main exposure of interest. Potential risk factors entered into the multivariable regression model were those that showed clinical relevance or showed a univariate relationship with pregnancy outcomes. The included variables were carefully selected based on the number of events available to ensure the stability of the regression equation. Adjusted odds ratio (OR) and their $95 \%$ confidence interval $(\mathrm{CI})$ were calculated to analyze the independent effect of immediate and delayed FET on the pregnancy outcomes.

Statistical significance was set at $P<0.05$. All statistical analyses were performed using IBM SPSS 26.0 (IBM Corp., Armonk, NY, USA).

\section{Results}

Potential risk factors between immediate and delayed FET groups in the entire and propensity score-matched cohort A total of 2128 HOR patients adopting a freeze-all strategy underwent their first IVF/ICSI cycle (Fig. 1). The immediate and delayed FET groups consisted of 1130 and 998 patients, respectively. Patients' and IVF characteristics in the immediate and delayed FET groups, which were the

Table 3 Pregnancy outcomes of immediate $\mathrm{FET}^{\mathbf{a}}$ and delayed FET ${ }^{\mathbf{b}}$ groups in the entire cohort and propensity score-matched cohort

\begin{tabular}{|c|c|c|c|c|c|c|}
\hline \multirow[t]{2}{*}{$\begin{array}{l}\text { Pregnancy } \\
\text { outcomes }\end{array}$} & \multicolumn{2}{|l|}{$\begin{array}{l}\text { Entire cohort } \\
(n=2128)\end{array}$} & \multirow[t]{2}{*}{$\begin{array}{l}P \text { - } \\
\text { value }\end{array}$} & \multicolumn{2}{|c|}{$\begin{array}{l}\text { Propensity score-matched cohort } \\
(n=1366)\end{array}$} & \multirow[t]{2}{*}{$\begin{array}{l}P \text { - } \\
\text { value }\end{array}$} \\
\hline & $\begin{array}{l}\text { Immediate } \mathrm{FET}^{\mathrm{a}} \\
(n=1130)\end{array}$ & $\begin{array}{l}\text { Delayed } \mathrm{FET}^{\mathrm{b}} \\
(n=998)\end{array}$ & & $\begin{array}{l}\text { Immediate } \mathrm{FET}^{\mathrm{a}} \\
(n=683)\end{array}$ & $\begin{array}{l}\text { Delayed } \mathrm{FET}^{\mathrm{b}} \\
(n=683)\end{array}$ & \\
\hline Clinical pregnancy & $61 \%$ & $63.4 \%$ & 0.244 & $413(60.5)$ & $434(63.5)$ & 0.242 \\
\hline Spontaneous abortion & $12.6 \%$ & $10.1 \%$ & 0.065 & 79 (11.6) & $84(12.3)$ & 0.676 \\
\hline Live birth & $49.9 \%$ & $49.2 \%$ & 0.743 & $328(48.0)$ & $337(49.3)$ & 0.626 \\
\hline
\end{tabular}

Abbreviations: FET, frozen embryo transfer

${ }^{\mathrm{a}}$ Immediate FET and ${ }^{\mathrm{b}}$ Delayed FET means FET took place either within the first menstrual cycle following oocyte retrieval or afterwards. Data are presented as number (\%) 
Table 4 Multivariable logistic regression analysis on potential risk factors for pregnancy outcomes in $\mathrm{GnRH}$-ant protocol $(n=918)$

\section{Potential risk factor variables}

Clinical pregnancy

FET timing (Immediate FET $^{\mathrm{a}}$ versus delayed $\mathrm{FET}^{\mathrm{b}}$ )

Age $(35-37$ versus $\leq 34)$

Age $(\geq 38$ versus $\leq 34)$

BMI (< 18.5 versus 18.5-24.9)

BMI ( $\geq 25$ versus $18.5-24.9$ )

Trigger type (GnRH-a trigger versus HCG trigger)

Trigger type (Double trigger versus HCG trigger)

Insemination method (ICSI versus IVF)

Number of embryo transferred (2 versus 1)

Embryo stage (blastocyst versus cleavage)

Top or good quality embryo transfer (yes versus no)

Tubal factor (yes versus no)

Ovulatory disorder (yes versus no)

Male factor (yes versus no)

Unexplained factor (yes versus no)

Spontaneous abortion

FET timing (Immediate FET ${ }^{\mathrm{a}}$ versus delayed $\mathrm{FET}^{\mathrm{b}}$ )

Age $(35-37$ versus $\leq 34)$

Age $(\geq 38$ versus $\leq 34)$

BMI (< 18.5 versus 18.5-24.9)

BMI ( $\geq 25$ versus 18.5-24.9)

Trigger type (GnRH-a trigger versus HCG trigger)

Trigger type (Double trigger versus HCG trigger)

Insemination method (ICSI versus IVF)

Number of embryo transferred (2 versus 1)

Embryo stage (blastocyst versus cleavage)

Top or good quality embryo transfer (yes versus no)

Tubal factor (yes versus no)

Ovulatory disorder (yes versus no)

Male factor (yes versus no)

Unexplained factor (yes versus no)

Live birth

FET timing (Immediate FET $^{\mathrm{a}}$ versus delayed FET ${ }^{\mathrm{b}}$ )

Age $(35-37$ versus $\leq 34)$

Age $(\geq 38$ versus $\leq 34)$

BMI ( $<18.5$ versus 18.5-24.9)

BMI ( $\geq 25$ versus 18.5-24.9)

Trigger type (GnRH-a trigger versus HCG trigger)

Trigger type (Double trigger versus HCG trigger)

Insemination method (ICSI versus IVF)

Number of embryo transferred (2 versus 1)

Embryo stage (blastocyst versus cleavage)

Top or good quality embryo transfer (yes versus no)
Adjusted OR* $(95 \% \mathrm{Cl})$

$P$-value

$\begin{array}{ll}0.956(0.721-1.267) & \mathrm{NS} \\ 0.798(0.502-1.269) & \mathrm{NS} \\ 0.386(0.210-0.708) & P<0.01 \\ 0.781(0.424-1.440) & \mathrm{NS} \\ 0.981(0.731-1.315) & \mathrm{NS} \\ 1.229(0.891-1.695) & \mathrm{NS} \\ 2.560(1.143-5.737) & P<0.05 \\ 1.006(0.696-1.453) & \mathrm{NS} \\ 1.726(1.040-2.865) & P<0.05 \\ 3.062(1.871-5.011) & P<0.001 \\ 0.984(0.647-1.497) & \mathrm{NS} \\ 1.043(0.745-1.461) & \mathrm{NS} \\ 1.242(0.909-1.695) & \mathrm{NS} \\ 1.012(0.720-1.423) & \mathrm{NS} \\ 1.268(0.622-2.585) & \mathrm{NS}\end{array}$

0.645 (0.430-0.966)

$P<0.05$

$1.202(0.620-2.329)$

NS

$1.511(0.696-3.280)$

NS

$0.716(0.245-2.095)$

NS

1.400 (0.934-2.100)

NS

0.907 (0.570-1.445)

NS

$1.408(0.613-3.232)$

NS

$1.274(0.756-2.144)$

NS

1.036 (0.553-1.943)

NS

1.537 (0.830-2.845)

NS

0.971 (0.538-1.753)

NS

0.847 (0.550-1.389)

NS

1.583 (1.017-2.465)

$P<0.05$

$0.793(0.483-1.304)$

NS

$0.978(0.353-2.710)$

NS

$1.220(0.925-1.608)$

NS

$0.761(0.479-1.210)$

NS

$0.249(0.121-0.514)$

$P<0.001$

0.883 (0.484-1.609)

NS

0.798 (0.599-1.063)

NS

$1.250(0.916-1.707)$

NS

1.867 (0.951-3.664)

NS

0.939(0.655-1.347)

NS

1.637 (1.027-2.610)

$P<0.05$

2.409 (1.533-3.785)

$P<0.001$

$1.052(0.701-1.580)$ 
Table 4 Multivariable logistic regression analysis on potential risk factors for pregnancy outcomes in GnRH-ant protocol $(n=918)$ (Continued)

\begin{tabular}{lll}
\hline Potential risk factor variables & Adjusted OR* $\mathbf{9 5 \% ~ C l )}$ & $P$-value \\
\hline Tubal factor (yes versus no) & $1.144(0.825-1.588)$ & NS \\
Ovulatory disorder (yes versus no) & $0.994(0.734-1.345)$ & NS \\
Male factor (yes versus no) & $1.154(0.827-1.611)$ & NS \\
Unexplained factor (yes versus no) & $1.232(0.619-2.450)$ & NS \\
\hline
\end{tabular}

Abbreviations: BMI, body mass index; FET, frozen embryo transfer; GnRH-a, gonadotrophin-releasing hormone agonist; GnRH-ant, gonadotrophin-releasing hormone antagonist; IVF, in vitro fertilization; ICSI, intracytoplasmic sperm injection; OR, odds ratio; Cl, confidence interval; NS, not significant

${ }^{a}$ Immediate FET and ${ }^{b}$ delayed FET means FET took place either within the first menstrual cycle following oocyte retrieval or afterwards. ${ }^{*}$ Using the multivariable logistic regression and adjusting for maternal age, BMl, insemination method, trigger type, number of embryo transferred, embryo stage, top or good quality embryo transfer, and cause of infertility

potential risk factors, are presented in Table 1. Before matching, the distribution of these risk factors were not absolutely balanced. The distribution of the $\mathrm{COH}$ protocol, number of embryos transferred, embryo stage, and multiple pregnancies were significantly different between the two groups $(P<0.05)$. No significant differences were found in maternal age, body mass index (BMI), insemination method, top or good quality embryo transfer, and infertility causes $(P>0.05)$. We obtained 1366 patients by PSM, and all potential risk factors and pregnancy outcomes of the multiple pregnancies were balanced and comparable (Table 1).

\section{Multivariable logistic regression analysis on FET timing and potential risk factors for pregnancy outcomes in the entire before-matching cohort}

Multivariable logistic regression analysis on the entire before-matching cohort demonstrated no statistical differences on pregnancy outcomes between the immediate and delayed FET groups [CPR, adjusted odd ratio (OR), 0.939, 95\% confidence interval (CI), 0.781-1.129; SAR, adjusted OR, 0.831, 95\% Cl, 0.628-1.098; LBR, adjusted OR, 1.056, 95\% Cl, 0.883-1.263] $(P>0.05)$, which were adjusted for maternal age, $\mathrm{BMI}, \mathrm{COH}$ protocol, insemination method, number of embryos transferred, embryo stage, trigger type, top or good quality embryo transfer, and cause of infertility (Table 2).

\section{Immediate versus delayed FET cycles on pregnancy outcomes in the entire cohort and propensity score- matched cohort}

The CPR (entire cohort, $61.0 \%$ versus $63.4 \%$; PSM cohort, $60.5 \%$ versus $63.5 \%$ ), SAR (entire cohort, $12.6 \%$ versus 10.1\%; PSM cohort, $11.6 \%$ versus $12.3 \%$ ), and LBR (entire cohort, 49.9\% versus 49.2\%; PSM cohort, $48.0 \%$ versus $49.3 \%$ ) had no significant differences between the immediate and delayed FET groups in the entire cohort and PSM cohort $(P>0.05)$ (Table 3$)$.

Differences between the groups were evaluated by $\chi^{2}$ test.
Subgroup analysis on immediate versus delayed FET on pregnancy outcomes in different $\mathrm{COH}$ protocol cohorts To investigate the effect of FET timing on pregnancy outcomes in different $\mathrm{COH}$ protocols, multivariable logistic regression were performed on each $\mathrm{COH}$ protocol cohort, including 918 patients in the antagonist protocol, 1000 patients in the short-acting GnRH-a long protocol, and 210 patients in the long-acting $\mathrm{GnRH}$-a long protocol. Multivariable logistic regression analysis demonstrated no statistical differences on pregnancy outcomes between the immediate and delayed FET groups in the short acting and long acting GnRH-a long protocols $(P>0.05)$. However, the SAR of the immediate FET group was lower than that of the delayed FET group in the GnRH-ant protocol (adjusted for maternal age, BMI, trigger type, insemination method, embryo stage, number of embryos transferred, top or good quality embryo transfer, and cause of infertility) (adjusted OR, 0.645, 95\% CI, 0.430-0.966) $(P<0.05)$. No significant differences were found on CPRs and LBRs in the GnRH-ant protocol $(P>0.05)$ (Tables $4,5,6)$.

\section{Discussion}

In clinical work, the timing of when to start the FET cycle after $\mathrm{COH}$ is controversial, especially for patients with $\mathrm{HOR}$ who are most affected by $\mathrm{COH}$. It is known that in the $\mathrm{COH}$ process, increased levels of supraphysiological steroid hormones and premature progesterone affects the gene expression and immune environment of the endometrium, which alters the embryoendometrium asynchrony and negatively affects endometrial receptivity, reducing the CPR and $\operatorname{LBR}[5,21]$. Moreover, the secretory activity factors produced by the residual luteal cysts derived from $\mathrm{COH}$ last longer after oocyte retrieval in HOR patients. However, no study has investigated the specific duration of these adverse effects. To avoid this concern, some clinicians recommend the conservative scheme which is to start the FET cycle at the second or third withdrawal bleeding after oocyte retrieval. This prolongs the IVF treatment period and increase the anxiety of patients who had experienced 
Table 5 Multivariable logistic regression analysis on risk factors for pregnancy outcomes in short-acting GnRH-a long protocol $(n=$ 1000)

\begin{tabular}{|c|c|c|}
\hline Potential risk factor variables & Adjusted $\mathrm{OR}^{*}(95 \% \mathrm{Cl})$ & $P$-value \\
\hline \multicolumn{3}{|l|}{ Clinical pregnancy } \\
\hline FET timing (Immediate FET $^{a}$ versus delayed FET ${ }^{\mathrm{b}}$ ) & $0.985(0.751-1.294)$ & NS \\
\hline Age $(35-37$ versus $\leq 34)$ & $0.987(0.636-1.530)$ & NS \\
\hline Age ( $\geq 38$ versus $\leq 34)$ & $0.414(0.194-0.879)$ & $P<0.05$ \\
\hline $\mathrm{BMI}(<18.5$ versus $18.5-24.9)$ & $1.391(0.792-2.442)$ & NS \\
\hline $\mathrm{BMI}(\geq 25$ versus $18.5-24.9)$ & $1.278(0.918-1.778)$ & NS \\
\hline Insemination method (ICSI versus IVF) & $0.727(0.508-1.040)$ & NS \\
\hline Number of embryo transferred (2 versus 1) & $1.401(0.911-2.155)$ & NS \\
\hline Embryo stage (blastocyst versus cleavage) & $2.318(1.595-3.370)$ & $P<0.001$ \\
\hline Top or good quality embryo transfer (yes versus no) & $1.148(0.824-1.598)$ & NS \\
\hline Tubal factor (yes versus no) & $1.109(0.767-1.603)$ & NS \\
\hline Ovulatory disorder (yes versus no) & $0.934(0.634-1.375)$ & NS \\
\hline Male factor (yes versus no) & $1.229(0.849-1.780)$ & NS \\
\hline Unexplained factor (yes versus no) & $2.101(0.888-4.971)$ & NS \\
\hline \multicolumn{3}{|l|}{ Spontaneous abortion } \\
\hline FET timing (Immediate FET $^{a}$ versus delayed FET ${ }^{b}$ ) & $1.311(0.826-2.080)$ & NS \\
\hline Age (35-37 versus $\leq 34)$ & $1.202(0.604-2.392)$ & NS \\
\hline Age ( $\geq 38$ versus $\leq 34$ ) & $1.047(0.297-3.699)$ & NS \\
\hline $\mathrm{BMI}(<18.5$ versus $18.5-24.9)$ & $1.246(0.506-3.067)$ & NS \\
\hline $\mathrm{BMI}(\geq 25$ versus $18.5-24.9)$ & $2.693(1.681-4.314)$ & $P<0.001$ \\
\hline Insemination method (ICSI versus IVF) & $1.069(0.567-2.015)$ & NS \\
\hline Number of embryo transferred (2 versus 1) & $0.856(0.433-1.693)$ & NS \\
\hline Embryo stage (blastocyst versus cleavage) & $0.853(0.470-1.547)$ & NS \\
\hline Top or good quality embryo transfer (yes versus no) & $1.380(0.764-2.495)$ & NS \\
\hline Tubal factor (yes versus no) & $0.439(0.229-0.841)$ & $P<0.05$ \\
\hline Ovulatory disorder (yes versus no) & $0.863(0.459-1.621)$ & NS \\
\hline Male factor (yes versus no) & $0.349(0.173-0.706)$ & $P<0.01$ \\
\hline Unexplained factor (yes versus no) & $0.225(0.046-1.102)$ & NS \\
\hline \multicolumn{3}{|l|}{ Live birth } \\
\hline FET timing (Immediate FET $^{a}$ versus delayed FET ${ }^{\mathrm{b}}$ ) & $0.929(0.714-1.209)$ & NS \\
\hline Age (35-37 versus $\leq 34)$ & $0.968(0.634-1.478)$ & NS \\
\hline Age $(\geq 38$ versus $\leq 34)$ & $0.430(0.195-0.949)$ & $P<0.05$ \\
\hline $\mathrm{BMI}(<18.5$ versus $18.5-24.9)$ & $1.263(0.743-2.150)$ & NS \\
\hline $\mathrm{BMI}(\geq 25$ versus $18.5-24.9)$ & $0.856(0.625-1.173)$ & NS \\
\hline Insemination method (ICSI versus IVF) & $0.797(0.562-1.130)$ & NS \\
\hline Number of embryo transferred (2 versus 1) & $1.362(0.913-2.033)$ & NS \\
\hline Embryo stage (blastocyst versus cleavage) & $2.283(1.613-3.231)$ & $P<0.001$ \\
\hline Top or good quality embryo transfer (yes versus no) & $1.134(0.820-1.569)$ & NS \\
\hline Tubal factor (yes versus no) & $1.458(1.017-2.091)$ & $P<0.05$ \\
\hline Ovulatory disorder (yes versus no) & $0.920(0.631-1.341)$ & NS \\
\hline Male factor (yes versus no) & $1.581(1.105-2.262)$ & $P<0.05$ \\
\hline Unexplained factor (yes versus no) & $2.914(1.306-6.500)$ & $P<0.01$ \\
\hline
\end{tabular}

Abbreviations: BMI, body mass index; FET, frozen embryo transfer; GnRH-a, gonadotrophin-releasing hormone agonist; IVF, in vitro fertilization; ICSI, intracytoplasmic sperm injection; OR, odds ratio; $\mathrm{Cl}$, confidence interval; NS, not significant

${ }^{a}$ Immediate FET and ${ }^{b}$ delayed FET means FET took place either within the first menstrual cycle following oocyte retrieval or afterwards

*Using the multivariable logistic regression and adjusting for maternal age, BMl, insemination method, number of embryo transferred, embryo stage, top or good quality embryo transfer, and cause of infertility 
Table 6 Multivariable logistic regression analysis on risk factors for pregnancy outcomes in long-acting GnRH-a long protocol

\begin{tabular}{|c|c|c|}
\hline Potential risk factor variables & Adjusted $\mathrm{OR}^{*}(95 \% \mathrm{Cl})$ & $P$-value \\
\hline \multicolumn{3}{|l|}{ Clinical pregnancy } \\
\hline FET timing (Immediate $\mathrm{FET}^{\mathrm{a}}$ versus delayed FET ${ }^{\mathrm{b}}$ ) & $0.607(0.322-1.147)$ & NS \\
\hline Age $(35-37$ versus $\leq 34)$ & $1.218(0.471-3.150)$ & NS \\
\hline Age $(\geq 38$ versus $\leq 34)$ & $0.810(0.1052-6.257)$ & NS \\
\hline $\mathrm{BMI}(<18.5$ versus $18.5-24.9)$ & $0.980(0.293-3.277)$ & NS \\
\hline BMI ( $\geq 25$ versus $18.5-24.9)$ & $1.525(0.715-3.256)$ & NS \\
\hline Insemination method (ICSI versus IVF) & $1.151(0.463-2.862)$ & NS \\
\hline Top or good quality embryo transfer (yes versus no) & $8.805(2.515-30.827)$ & $P<0.01$ \\
\hline Embryo stage (blastocyst versus cleavage) & $1.425(1.163-5.056)$ & $P<0.05$ \\
\hline Tubal factor (yes versus no) & $2.454(0.924-6.518)$ & NS \\
\hline Ovulatory disorder (yes versus no) & $0.840(0.229-3.085)$ & NS \\
\hline Male factor (yes versus no) & $2.844(1.147-7.051)$ & $P<0.05$ \\
\hline Unexplained factor (yes versus no) & $1.335(0.204-8.721)$ & NS \\
\hline Spontaneous abortion & Adjusted $\mathrm{OR}^{\#}(95 \% \mathrm{Cl})$ & \\
\hline FET timing (Immediate FET ${ }^{a}$ versus delayed FET ${ }^{b}$ ) & $0.380(0.143-1.010)$ & NS \\
\hline Age $(35-37$ versus $\leq 34)$ & $0.409(0.088-1.878)$ & NS \\
\hline Age $(\geq 38$ versus $\leq 34)$ & - & NS \\
\hline $\mathrm{BMI}(<18.5$ versus $18.5-24.9)$ & $0.544(0.066-4.491)$ & NS \\
\hline BMI ( $\geq 25$ versus $18.5-24.9)$ & $1.914(0.759-4.827)$ & NS \\
\hline Top or good quality embryo transfer (yes versus no) & $0.836(0.207-3.375)$ & NS \\
\hline Live birth & Adjusted $\mathrm{OR}^{*}(95 \% \mathrm{Cl})$ & $P$-value \\
\hline FET timing (Immediate FET ${ }^{a}$ versus delayed FET ) & $0.995(0.543-1.823)$ & NS \\
\hline Age $(35-37$ versus $\leq 34)$ & $2.034(0.800-5.168)$ & NS \\
\hline Age $(\geq 38$ versus $\leq 34)$ & $1.991(0.248-15.991)$ & NS \\
\hline $\mathrm{BMI}(<18.5$ versus $18.5-24.9)$ & $1.290(0.396-4.195)$ & NS \\
\hline BMI ( $\geq 25$ versus $18.5-24.9)$ & $1.034(0.507-2.111)$ & NS \\
\hline Insemination method (ICSI versus IVF) & $1.422(0.607-3.334)$ & NS \\
\hline Top or good quality embryo transfer (yes versus no) & $13.211(2.621-66.596)$ & $P<0.01$ \\
\hline Embryo stage (blastocyst versus cleavage) & $2.339(1.186-4.614)$ & $P<0.05$ \\
\hline Tubal factor (yes versus no) & $2.025(0.842-4.868)$ & NS \\
\hline Ovulatory disorder (yes versus no) & $0.552(0.148-2.057)$ & NS \\
\hline Male factor (yes versus no) & $1.470(0.658-3.286)$ & NS \\
\hline Unexplained factor (yes versus no) & $0.397(0.054-2.935)$ & NS \\
\hline
\end{tabular}

Abbreviations: BMI, body mass index; FET, frozen embryo transfer; GnRH-a, gonadotrophin-releasing hormone agonist; IVF, in vitro fertilization; ICSI, intracytoplasmic sperm injection; $\mathrm{OR}$, odds ratio; $\mathrm{Cl}$, confidence interval; $\mathrm{NS}$, not significant

${ }^{\mathrm{a}}$ Immediate FET and ${ }^{\mathrm{b}}$ delayed FET means FET took place either within the first menstrual cycle following oocyte retrieval or afterwards

*Using the multivariable logistic regression and adjusting for maternal age, BMI, insemination method, trigger type, embryo stage, top or good quality embryo transfer, and cause of infertility

\# Using the multivariable logistic regression and adjusting for maternal age, BMI, top or good quality embryo transfer

"-" means that the number of miscarriages in the group over 38 years old is 0 .

infertility for many years, increasing their mental and economic loses and affecting their pregnancy outcomes $[10,22]$. Lattes et al. reported that there were no differences on the first FET and subsequent FET cycles after oocyte retrieval [23]. A retrospective study by Huang et al. demonstrated that immediate FET was associated with a higher live birth than delayed FET [24], and meta-analysis indicated that immediate FET was not associated with negative pregnancy outcomes [25]. In our retrospective study, we only selected the HOR population who adopted HRT as the endometrial preparation protocol in the past five years and found no significant differences for CPR, SAR, and LBR between the immediate and delayed FET groups. We believe it is not 
accurate to assume that $\mathrm{COH}$ will still affect endometrial receptivity in the first withdrawal bleeding cycle after oocyte retrieval and that it is not necessary for the HOR population to wait for several menstrual cycles to begin FET after the freeze-all strategy.

In the process of $\mathrm{COH}$, different $\mathrm{GnRH}$ analogues have different degrees and properties of inhibition effects on hypothalamic-pituitary-ovarian axis, and they also have different effects on the corpus luteum [26], which might have an impact on endometrial receptivity and pregnancy outcomes [27]. A retrospective study showed that immediate FET had similar CPR to delayed FET in patients with GnRH-ant protocol [13], which is in agreement with our subgroup results. However, our results are contrary to a population-based study on the short-acting GnRH-a long protocol, which found that delayed FET was better for pregnancy outcomes. They believed that the initial flare up effect of short acting GnRH-a during the downregulation period caused an early rise of progesterone, which affected the outcomes in the immediate FET cycle [11]. However, a limitation of that study was the small sample size of 67 patients in the immediate FET group and 62 in the delayed FET group. In this study, 1000 patients with short acting GnRH-a long protocol were studied (434 in immediate FET group and 566 in delayed FET group), and we found that the timing of FET did not affect pregnancy outcomes in the short-acting GnRH-a long protocol. However, in the GnRH-ant protocol, we found that the SAR in the delayed FET group was significantly higher than that in the immediate FET group. Among all the $\mathrm{COH}$ protocols, the $\mathrm{GnRH}$-ant protocol has the shortest treatment period, where oocyte retrieval takes place after a mean of 8-10 days of ovarian stimulation; the short-acting $\mathrm{GnRH}$-a long protocol requires 14 days of down-regulation on that basis; while the long-acting GnRH-a long protocol needs a down-regulation of more than 20 days. Therefore, the immediate FET cycle in the $\mathrm{GnRH}$-ant protocol can result in the shortest treatment period. Psychological factors can lead to infertility and spontaneous abortion, and its potential impact on neuroendocrine and immune changes could affect early pregnancy risk $[28,29]$. We considered that longer treatment periods could result in increased patient anxiety, causing the increase in the SAR. It is worth mentioning that one study has shown that residual luteal cysts may increase the expression of relaxin in circulation [30], which is related to endometrial angiogenesis and prevents recurrent abortion [31]. Therefore, the effects of residual luteal cysts in immediate FET cycles that we had previously worried about may be beneficial to endometrial receptivity and pregnancy outcomes.

The limitation of this study lies in the retrospective nature as well as the possibility of unmeasured confounding factors such as smoking habits and alcohol consumption. Although we have obtained many cases with HOR and made the immediate and delayed FET groups comparable by PSM, we have lost 762 cases without successful matching in this process. In addition, we do not know whether these cases will affect the actual situation. Notably, the pregnancy outcomes we have studied were not the only endpoint, as other obstetric outcomes and neonatal outcomes should also be recorded. Moreover, the self-selection of patients into immediate or delayed FET remains a major limitation of the study. We believe that further prospective, randomized, and controlled studies are needed to confirm these results.

In summary, this study indicated that FET timing might not affect pregnancy outcomes in high responder patients undergoing freeze-all cycles. Immediate FET might be associated with lower odds of spontaneous abortion.

\section{Conclusion}

The pregnancy outcomes of immediate FET were no different to delayed FET in high responder patients undergoing freeze-all cycles.

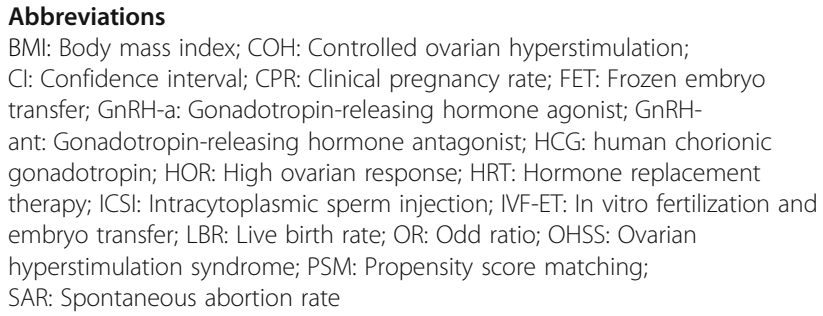

\section{Acknowledgments}

Not applicable.

\section{Authors' contributions}

D.L. and X.X.W. conceived and designed the study. N.Z., N.N.Z., Y.Z.G. performed the data collection and analysis. D.L., X.X.W., N.Z. and Y.Z.G. wrote the paper. All authors read and approved the final manuscript.

\section{Funding}

This work was supported by the National Natural Science Foundation of China (grant number 82071607 and 81671423), the National Key Research and Development Program of China (grant number 2018YFC1003100), Liaoning Revitalization Talents Program (grant number XLYC1907071), Liaoning Provincial Key Research and Development Program (grant number 2018225090), the Fok Ying Tung Education Foundation (grant number 151039)

\section{Availability of data and materials}

The datasets generated and analysed during the current study were used under license for the current study, and so are not publicly available.

\section{Declarations}

Ethics approval and consent to participate

All patients gave written informed consent, and the study was approved by the Institutional Review Board at Shengjing hospital of China Medical University (reference number 2020PS006F). 


\section{Consent for publication}

Not applicable.

\section{Competing interests}

The authors declare that they have no competing interests.

\section{Author details}

${ }^{1}$ Center of Reproductive Medicine, Shengjing Hospital of China Medical University, Shenyang 110004, China. 'Department of Obstetrics and Gynecology, Shengjing Hospital of China Medical University, Shenyang 110004, China

Received: 17 November 2020 Accepted: 28 May 2021

Published online: 28 June 2021

\section{References}

1. Chen CH, Zhang X, Barnes R, Confino E, Milad M, Puscheck E, et al. Relationship between peak serum estradiol levels and treatment outcome in in vitro fertilization cycles after embryo transfer on day 3 or day 5 . Fertil Steril. 2003:80(1):75-9. https://doi.org/10.1016/S0015-0282(03)00504-1.

2. Junovich G, Mayer Y, Azpiroz A, Daher S, Iglesias A, Zylverstein C, et al. Ovarian stimulation affects the levels of regulatory endometrial NK cells and angiogenic cytokine VEGF. Am J Reprod Immunol. 2011;65(2):146-53. https://doi.org/10.1111/j.1600-0897.2010.00892.x.

3. Haouzi D, Assou S, Mahmoud K, Tondeur S, Reme T, Hedon B, et al. Gene expression profile of human endometrial receptivity: comparison between natural and stimulated cycles for the same patients. Hum Reprod. 2009; 24(6):1436-45. https://doi.org/10.1093/humrep/dep039.

4. Venetis CA, Kolibianakis EM, Bosdou JK, Tarlatzis BC. Progesterone elevation and probability of pregnancy after IVF: a systematic review and metaanalysis of over 60000 cycles. Hum Reprod Update. 2013;19(5):433-57. https://doi.org/10.1093/humupd/dmt014.

5. Ozgur K, Berkkanoglu M, Bulut H, Isikli A, Coetzee K. Higher clinical pregnancy rates from frozen-thawed blastocyst transfers compared to fresh blastocyst transfers: a retrospective matched-cohort study. J Assist Reprod Genet. 2015;32(10):1483-90. https://doi.org/10.1007/s10815-0150576-1.

6. Practice Committee of the American Society for Reproductive Medicine. Electronic address. Prevention and treatment of moderate and severe ovarian hyperstimulation syndrome: a guideline. Fertil Steril 2016, 106(7): 1634-1647, DOl: https://doi.org/10.1016/j.fertnstert.2016.08.048.

7. Pelkonen S, Koivunen R, Gissler M, Nuojua-Huttunen S, Suikkari AM, HydenGranskog C, et al. Perinatal outcome of children born after frozen and fresh embryo transfer: the Finnish cohort study 1995-2006. Hum Reprod. 2010; 25(4):914-23. https://doi.org/10.1093/humrep/dep477.

8. Liu SY, Teng B, Fu J, Li X, Zheng Y, Sun XX. Obstetric and neonata outcomes after transfer of vitrified early cleavage embryos. Hum Reprod. 2013;28(8):2093-100. https://doi.org/10.1093/humrep/det104.

9. Pelkonen S, Hartikainen AL, Ritvanen A, Koivunen R, Martikainen H, Gissler $\mathrm{M}$, et al. Major congenital anomalies in children born after frozen embryo transfer: a cohort study 1995-2006. Hum Reprod. 2014;29(7):1552-7. https:// doi.org/10.1093/humrep/deu088.

10. Maas KH, Baker VL, Westphal LM, Lathi RB. Optimal timing of frozen embryo transfer after failed IVF attempt. Fertil Steril. 2008;90:\$285. https://doi.org/1 0.1016/j.fertnstert.2008.07.1101.

11. Volodarsky-Perel A, Eldar-Geva T, Holzer HE, Schonberger O, Reichman O, Gal M. Cryopreserved embryo transfer: adjacent or non-adjacent to failed fresh long GnRH-agonist protocol IVF cycle. Reprod BioMed Online. 2017; 34(3):267-73. https://doi.org/10.1016/j.rbmo.2016.11.013.

12. Hsieh YY, Chang CC, Tsai HD. Comparisons of different dosages of gonadotropin-releasing hormone $(\mathrm{GnRH})$ antagonist, short-acting form and single, half-dose, long-acting form of GnRH agonist during controlled ovarian hyperstimulation and in vitro fertilization. Taiwan J Obstet Gynecol. 2008;47(1):66-74. https://doi.org/10.1016/S1028-4 559(08)60057-1

13. Santos-Ribeiro S, Polyzos NP, Lan VT, Siffain J, Mackens S, Van Landuyt L, et al. The effect of an immediate frozen embryo transfer following a freezeall protocol: a retrospective analysis from two centres. Hum Reprod. 2016; 31(11):2541-8. https://doi.org/10.1093/humrep/dew194.

14. Papanikolaou EG, Pozzobon C, Kolibianakis EM, Camus M, Tournaye $H_{\text {, }}$ Fatemi HM, et al. Incidence and prediction of ovarian hyperstimulation syndrome in women undergoing gonadotropin-releasing hormone antagonist in vitro fertilization cycles. Fertil Steril. 2006;85(1):112-20. https:// doi.org/10.1016/j.fertnstert.2005.07.1292.

15. Magnusson A, Kallen K, Thurin-Kjellberg A, Bergh C. The number of oocytes retrieved during IVF: a balance between efficacy and safety. Hum Reprod. 2018;33(1):58-64. https://doi.org/10.1093/humrep/dex334

16. Hamdine O, Eijkemans MJ, Lentjes EW, Torrance HL, Macklon NS, Fauser BC, et al. Ovarian response prediction in $\mathrm{GnRH}$ antagonist treatment for IVF using anti-Mullerian hormone. Hum Reprod. 2015;30(1):170-8. https://doi. org/10.1093/humrep/deu266.

17. Scheinhardt MO, Lerman T, Konig IR, Griesinger G. Performance of prognostic modelling of high and low ovarian response to ovarian stimulation for IVF. Hum Reprod. 2018;33(8):1499-505. https://doi.org/10.1 093/humrep/dey236.

18. Alpha Scientists in Reproductive M, Embryology ESIGo. The Istanbul consensus workshop on embryo assessment: proceedings of an expert meeting. Hum Reprod. 2011;26(6):1270-83.

19. Gardner DK, Surrey E, Minjarez D, Leitz A, Stevens J, Schoolcraft WB. Single blastocyst transfer: a prospective randomized trial. Fertil Steril. 2004;81(3): 551-5. https://doi.org/10.1016/j.fertnstert.2003.07.023.

20. Zegers-Hochschild F, Adamson GD, de Mouzon J, Ishihara O, Mansour R, Nygren $\mathrm{K}$, et al. International Committee for Monitoring Assisted Reproductive Technology (ICMART) and the World Health Organization (WHO) revised glossary of ART terminology, 2009. Fertil Steril. 2009;92(5): 1520-4.

21. Venetis CA, Kolibianakis EM, Bosdou JK, Lainas GT, Sfontouris IA, Tarlatzis BC, et al. Estimating the net effect of progesterone elevation on the day of hCG on live birth rates after IVF: a cohort analysis of 3296 IVF cycles. Hum Reprod. 2015;30(3):684-91. https://doi.org/10.1093/humrep/deu362.

22. Higgins $C$, Healey M, Jatkar S, Vollenhoven B. Interval between IVF stimulation cycle and frozen embryo transfer: is there a benefit to a delay between cycles? Aust N Z J Obstet Gynaecol. 2018;58(2):217-21. https://doi. org/10.1111/ajo.12696.

23. Lattes $K$, Checa MA, Vassena $R$, Brassesco M, Vernaeve $V$. There is no evidence that the time from egg retrieval to embryo transfer affects live birth rates in a freeze-all strategy. Hum Reprod. 2017;32(2):368-74. https:// doi.org/10.1093/humrep/dew306.

24. Huang J, Lu X, Xie Q, Lin J, Cai R, Kuang Y. Timing of frozen-thawed embryo transfer after controlled ovarian stimulation in a non-elective freeze-all policy. Ann Transl Med. 2019;7(23):752. https://doi.org/10.2103 7/atm.2019.11.74

25. Huang J, Lin J, Lu X, Cai R, Song N, Kuang Y. Delayed versus immediate frozen embryo transfer after oocyte retrieval: a systematic review and metaanalysis. J Assist Reprod Genet. 2020;37(8):1949-57. https://doi.org/10.1007/ s10815-020-01857-9.

26. Lambalk CB, Banga FR, Huirne JA, Toftager M, Pinborg A, Homburg R, et al. $\mathrm{GnRH}$ antagonist versus long agonist protocols in IVF: a systematic review and meta-analysis accounting for patient type. Hum Reprod Update. 2017; 23(5):560-79. https://doi.org/10.1093/humupd/dmx017.

27. Mao GH, Feng Z, He Y, Huang YR. Comparisons of the effects of long-acting and short-acting GnRH agonists on embryo quality, endometrial thickness and pregnancy rate in human in vitro fertilization. Arch Med Sci. 2014;10(1): 161-6. https://doi.org/10.5114/aoms.2014.40743.

28. Frazier T, Hogue CJR, Bonney EA, Yount KM, Pearce BD. Weathering the storm; a review of pre-pregnancy stress and risk of spontaneous abortion. Psychoneuroendocrinology. 2018;92:142-54. https://doi.org/10.1016/j. psyneuen.2018.03.001

29. Boivin J, Gameiro S. Evolution of psychology and counseling in infertility. Fertil Steril. 2015;104(2):251-9. https://doi.org/10.1016/j.fertnstert.2015.05.035.

30. Conrad KP, Baker VL. Corpus luteal contribution to maternal pregnancy physiology and outcomes in assisted reproductive technologies. Am J Physiol Regul Integr Comp Physiol. 2013;304(2):R69-72. https://doi.org/10.11 52/ajpregu.00239.2012.

31. Chen X, Man GCW, Liu Y, Wu F, Huang J, Li TC, et al. Physiological and pathological angiogenesis in endometrium at the time of embryo implantation. Am J Reprod Immunol. 2017;78(2):e12693. https://doi.org/1 $0.1111 /$ aji.12693

\section{Publisher's Note}

Springer Nature remains neutral with regard to jurisdictional claims in published maps and institutional affiliations. 